\title{
Caracterización morfológica de un lecho de recubrimiento de tabletas farmacéuticas mediante un algoritmo de visión artificial
}

\section{Morphological characterization of a pharmaceutical tablet coating bed using an artificial vision algorithm} Joel Jesús Alpízar-Castillo¹, Mac Arturo Murillo-Fernández²

Fecha de recepción: 3 de abril de 2020

Alpízar-Castillo, J.J; Murillo-Fernández, M.A. Caracterización morfológica de un lecho de recubrimiento de tabletas farmacéuticas mediante un algoritmo de visión artificial. Tecnología en Marcha. Vol. 34-3 Julio-Setiembre 2021. Pág 51-60.

doi) https://doi.org/10.18845/tm.v34i3.5032

1 Área Académica de Ingeniería Mecatrónica, Instituto Tecnológico de Costa Rica. Costa Rica. Correo electrónico: Joel.AlpizarCastillo@gmail.com (D) https://orcid.org/0000-0002-1238-4430

2 Escuela de Física, Instituto Tecnólogico de Costa Rica. Costa Rica. Correo electrónico: macarturo61@gmail.com 


\title{
Palabras clave
}

Morfología tabletas; recubrimiento de tabletas; sistema de visión artificial.

\section{Resumen}

La industria farmacéutica se encuentra en la búsqueda constante de métodos de optimización de sus procesos. En la presente investigación se desarrolló un sistema de visión que permite caracterizar morfológicamente el lecho de tabletas farmacéuticas en el proceso de recubrimiento. Como resultado, se diseñó un algoritmo de visión artificial que permite cuantificar las tabletas presentes en la imagen y determinar el área de cada una mediante el uso de la transformada de Hough y el filtro de Canny, partiendo de un modelo plano y estático del lecho de recubrimiento de tabletas farmacéuticas. Se obtuvo una aproximación de la posición angular de las tabletas farmacéuticas respecto a eje óptico de la cámara, según la cantidad de capas de tabletas en el lecho.

\section{Keywords}

Artificial vision system; tablet coating; tablet morphology.

\begin{abstract}
The pharmaceutical industry is constantly searching for methods to optimize its processes. In the present investigation was developed a vision system that allows to morphologically characterize the bed of pharmaceutical tablets during the coating process. As a result, an artificial vision algorithm was designed, which allows to quantify the tablets shown in the image and determine the area of each one, through the use of the Hough transformation and the Canny filter, based on a flat and static model of the coating bed of pharmaceutical tablets. An approximation of the angular position of the pharmaceutical tablets with respect to the optical axis of the camera was obtained, according to the number of layers of tablets in the coating bed.
\end{abstract}

\section{Definición del problema}

Un elemento clave en la dosificación de medicamentos por parte de industria farmacéutica son los encapsulados o "tabletas", cuyo proceso de fabricación, por lo general, implica una cobertura. El proceso anterior se realiza para cubrir algunas características del núcleo, como sabor, olor, textura y fragilidad, entre otros [1].

Esta cobertura se realiza en un tambor en el que se hacen girar las tabletas, dentro del cual una pistola dispersa una mezcla de recubrimiento pigmentado al lecho de tabletas. El color resultante y el grosor del recubrimiento se determinan, a priori, en función del tiempo que permanezcan las tabletas girando en el bombo o tambor de recubrimiento [2]. De este modo, la posición angular de las tabletas respecto al eje axial de la pistola tiene un papel importante en la eficiencia del proceso de recubrimiento, por lo que una cuantificación morfológica del acomodo de las tabletas permitiría optimizar el proceso por medio de modelos de simulación, para la implementación de sistemas automatizados en la industria farmacéutica, según las recomendaciones de la FDA, [3].

El control de calidad visual en estos procesos, por lo general es de naturaleza subjetiva, y requiere detener el proceso, extraer las muestras y analizar el aspecto del producto resultante (color, grosor de la capa, acabado, entre otros), por lo que la implementación de un método objetivo para el control de calidad sería de gran utilidad [4]. Doerr y sus colegas mencionan 
en [5] que es común el uso de tomografía micro-enfocada para analizar la calidad estructural y la homogeneidad de la muestra. A pesar de lo anterior, resultados experimentales sobre el cambio de color producto de la aspersión de las tabletas no se encontraron en la literatura hasta el trabajo de Murillo et. al. presentado en [1], [4] y [6].

Antes de proponer un sistema de control de calidad completo, es necesario determinar el comportamiento de una de las variables más importantes, la variación morfológica de las tabletas durante el proceso de recubrimiento, que incluye no solo el aumento de tamaño producto del recubrimiento, y los posibles daños superficiales, sino que también incluye los diferentes cambios de posición y forma, producto de la rotación en el bombo de recubrimiento, que deberían ser modelados y distinguidos por cualquier sistema de visión artificial.

En esta investigación, se propone un algoritmo de visión artificial que, partiendo de las imágenes fotográficas de un modelo estático simplificado lecho de recubrimiento del lecho de recubrimiento de tabletas, que permite caracterizar morfológicamente su distribución, contribuyendo en trabajos como el desarrollado en [6]. El desarrollo del algoritmo propuesto posibilita el desarrollo de en un sistema de visión artificial de alta velocidad que permitiría el análisis en tiempo real del comportamiento morfológico de las tabletas durante el proceso de cobertura.

\section{Antecedentes}

Como se menciona en [7], los procesos de recubrimiento de tabletas farmacéuticas han probado ser un desafío dentro del contexto del control de calidad industrial producto de la variabilidad producto de los recubrimientos por aspersión aleatoria. Los principales métodos para caracterizar las capas de recubrimiento se mencionan en [8], destacando: microscopía de fuerza atómica, microscopía láser confocal, espectroscopía fotoelectrónica de rayos X, resonancia paramagnética electrónica, espectroscopía infrarroja por transformada de Fourier, espectroscopía de ruptura inducida por láser y microscopía térmica, procesos que, si bien no son necesariamente destructivos, implican detener el proceso productivo para el análisis, lo que es contraproducente en procesos industriales automatizados [9].

Dentro de los esfuerzos por parte de la FDA por mejorar los procesos anteriormente descritos, surgen las guías ICH (International Council for Harmonization of Technical Requirements for Pharmaceuticals for Human Use), dada las evidentes oportunidades de mejora que presentan [10], ya que los procesos de control de calidad tradicionales implican la extracción y análisis de muestras [8], en lugar de poder analizar el proceso en tiempo real, como se propone en [7].

\section{Planteamiento de la solución}

\section{Descripción del modelo}

Se utilizó un modelado estático del lecho de recubrimiento de tabletas en el tambor durante el proceso de recubrimiento para simplificar su análisis, basado en la eventual utilización de una cámara de alta velocidad al momento de implementar el sistema propuesto. Para esto, se dispusieron las tabletas en una superficie plana, analizando tres casos independientes en cuanto a la cantidad de capas de tabletas (una, dos y tres capas), de modo que sea posible una generalización del algoritmo, independientemente del número de capas en el lecho de recubrimiento. 
Para la toma de las fotografías se utilizó una cámara Cognex 7200, con un lente de 16 mm, a una distancia de trabajo de $41 \mathrm{~cm}$ sobre un fondo plano y negro con una combinación de iluminación natural y fluorescente. Las imágenes utilizadas tienen unas dimensiones de 800 pixeles $\times 600$ pixeles.

\section{Descripción del algoritmo}

Para el análisis de las fotografías, es importante aclarar que, como la cámara toma imágenes en escala de grises, no fue necesaria su conversión a una imagen monocromática; además, debido a que se contó con una cantidad limitada de tabletas, las fotografías tomadas como muestra contaron con lechos de entre una y tres capas de tabletas. Así, en primera instancia se les realizó una transformación gamma a las fotografías para mejorar su calidad, de modo que su procesado fuera más eficiente. En las figuras 1.a y 2.a se ejemplifican las imágenes tomadas como base para la evaluación del algoritmo ante lechos con diferente número de capas de tabletas (una capa en la figura 1 y tres capas en la figura 3).

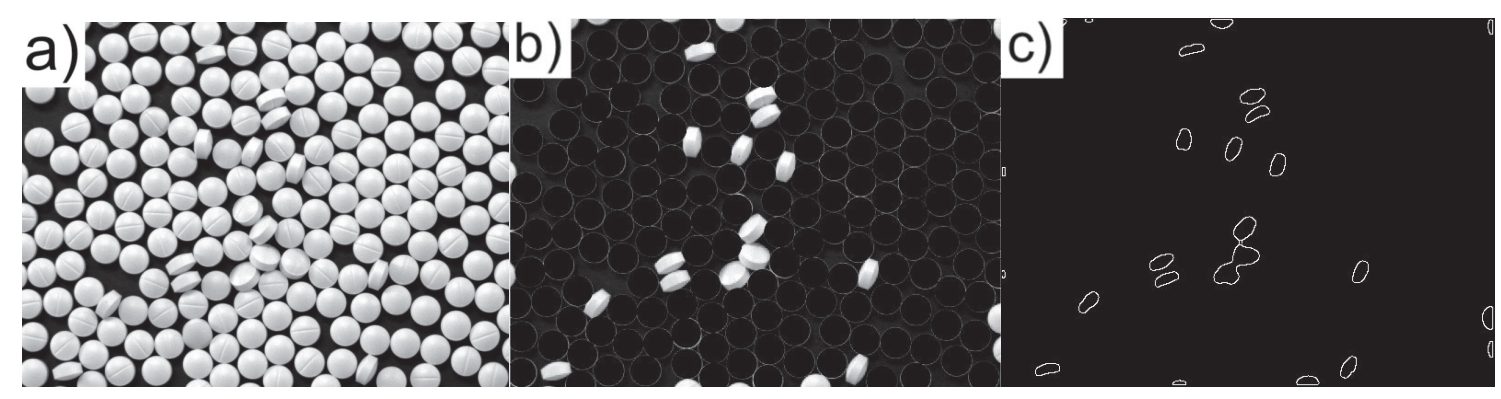

Figura 1. a) Imagen original con transformación gamma, una capa de tabletas. b) Imagen a la que se le eliminaron las tabletas completas, una capa de tabletas. c) Imagen con detección de contornos para las tabletas inclinadas, una capa de tabletas.

Para el análisis morfológico de la imagen se separaron las muestras en dos grandes conjuntos: las tabletas que se encuentra con su cara perpendicular a la cámara y aquellas que se encuentran de costado o con cierto ángulo de inclinación. Para el primer grupo, se modelaron las tabletas como círculos y se utilizó la transformada de Hough para la ubicación sus centros, almacenando sus radios en pixeles y eliminando las regiones de la imagen en las que se encontraban, insertando círculos negros con el centro y radio detectado para cada tableta, como se muestra en las figuras 1.b y 2.b.

Una vez eliminadas las tabletas perpendiculares de la imagen, para las tabletas que no se encontraban perpendiculares a la cámara, se utilizó un filtro de Canny como detector de contornos, con una umbralización y sensibilidad específicas, para identificarlas como elementos individuales y así determinar sus respectivas áreas, como se muestra en las figuras 1.c y 2.c.

La comparación de las áreas individuales de las tabletas, con el promedio de los círculos detectados, permitió obtener la distribución de las tabletas en el área perpendicular a la cámara. Nótese que, las áreas obtenidas se encuentran en número de pixeles, por lo que, fue necesaria la conversión a milímetros cuadrados, según las mediciones de las tabletas, tomando $7 \mathrm{~mm}$ como valor de referencia teórica para su diámetro. 


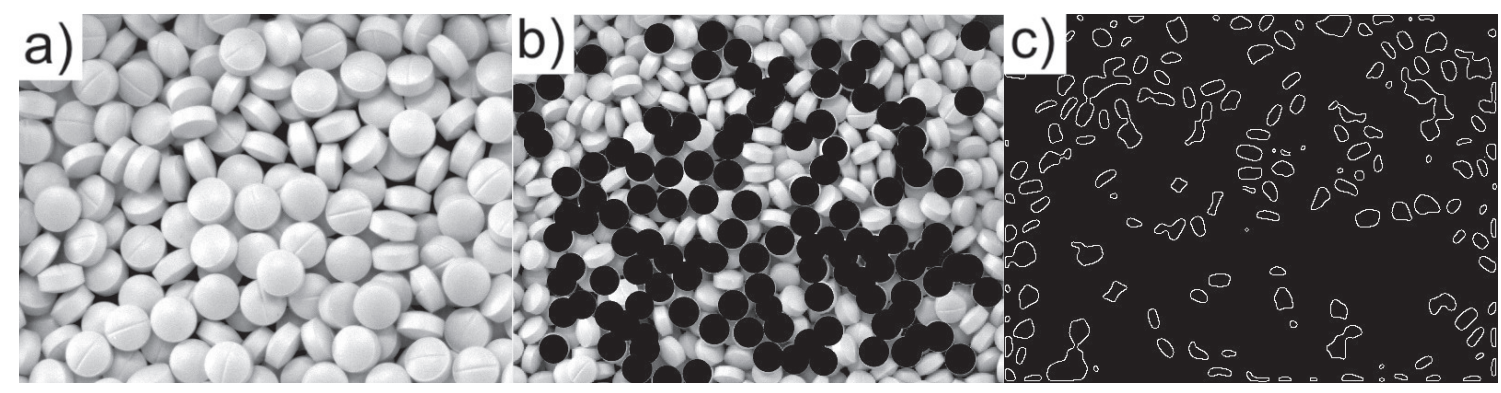

Figura 2. a) Imagen original con transformación gamma, tres capas de tabletas. b) Imagen a la que se le eliminaron las tabletas completas, tres capas de tabletas. c) Imagen con detección de contornos para las tabletas inclinadas, tres capas de tabletas.

\section{Resultados}

Al ejecutar el algoritmo, por la naturaleza de las funciones implementadas, se debieron ajustar los siguientes parámetros para obtener un funcionamiento idóneo del algoritmo. Se encontró experimentalmente que, una sensibilidad del $95 \%$ y un límite de umbralización de 150, se diferencian las tabletas del fondo de manera óptima. Por su parte, un rango de radios de 20 pixeles a 25 pixeles permitió identificar con mayor precisión las tabletas perpendiculares a la cámara.

Teniendo lo anterior en cuenta, se ejecutó el algoritmo con fotografías de una lecho con una, dos y tres capas de tabletas, permitiendo un conteo exitoso de las tabletas. Como es intuitivo, al incrementar el número de capas, la cantidad de tabletas perpendiculares a la cámara disminuye y su distribución se torna más compleja, dificultando el cálculo del área de las tabletas inclinadas, según puede apreciarse en la figura 2, donde se muestran las etapas para una fotografía de el lecho con tres capas de tabletas. Todas las fotografías utilizadas se muestran en el cuadro A.1 en la sección de Anexos.

Por cada prueba, se utilizaron cinco fotografías para el lecho de recubrimiento de tabletas con cada número de capas. De este modo, se obtuvo un promedio general de 222 tabletas por fotografía, y la distribución para cada número de capas se presenta en la figura 3.

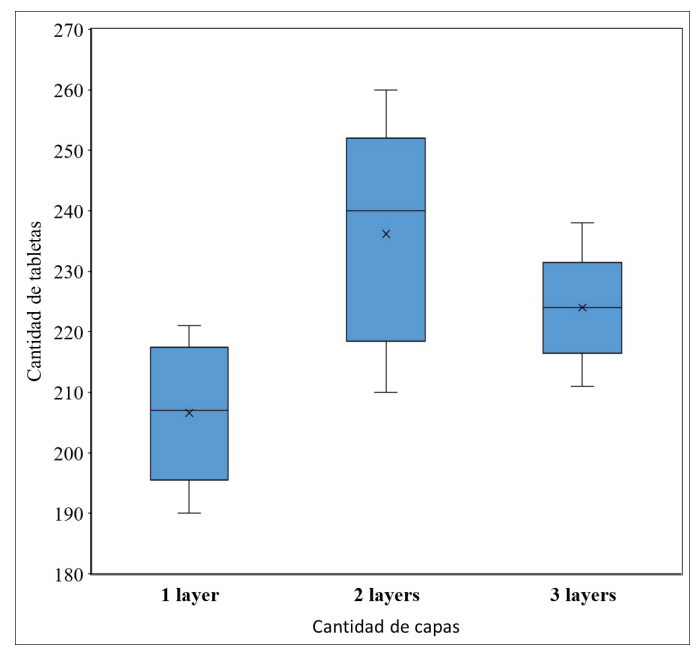

Figura 3. Distribución del conteo de tabletas por número de capas. 


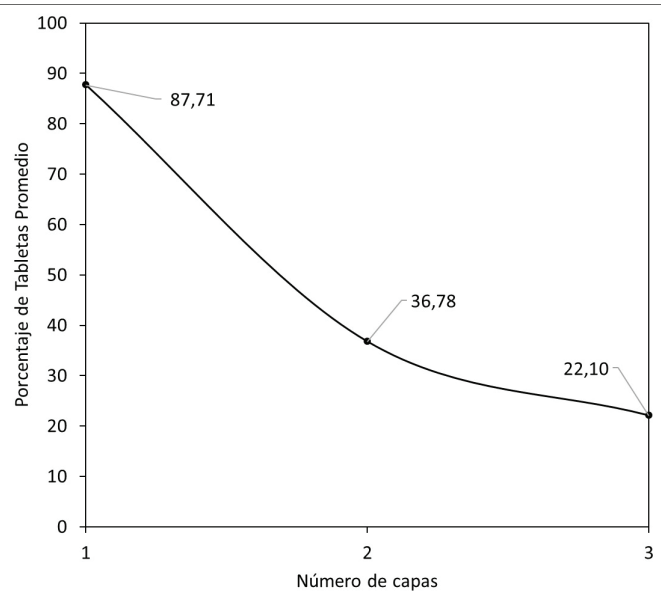

Figura 4. Número de tabletas perpendiculares en función del número de capas.

Del mismo modo, en el cuadro 1 se muestra el porcentaje promedio de tabletas por número de capas que se encuentran totalmente perpendiculares al eje óptico, al igual que el promedio del área de las tabletas perpendiculares con sus respectivos porcentajes de error. Este se calculó en función de un radio teórico de $7 \mathrm{~mm}$. Un mayor detalle en cuanto a las áreas promedio y su respectivo porcentaje de error, definido por la diferencia entre el área detectada por el algoritmo, y el área teórica de las tabletas, puede encontrarse en el cuadro A.2. en la sección de Anexos.

El algoritmo implementado se ejecutó en una computadora con un procesador Intel Core i5 de cuarta generación, con 16 GB de RAM, obteniendo tiempos de respuesta del algoritmo del orden de $1 \mathrm{~s}$ por imagen.

Cuadro 1. Datos promedio obtenidos por capa.

\begin{tabular}{|c|c|c|c|}
\hline $\begin{array}{c}\text { Número de } \\
\text { capas }\end{array}$ & $\begin{array}{c}\text { Porcentaje de tabletas } \\
\text { perpendiculares }\end{array}$ & $\begin{array}{c}\text { Área de las tabletas } \\
\text { perpendiculares }\end{array}$ & $\begin{array}{c}\text { Porcentaje de } \\
\text { error }\end{array}$ \\
\hline 1 & $87.7 \%$ & $36,4 \mathrm{~mm}^{2}$ & $5,4 \%$ \\
\hline 2 & $36.8 \%$ & $37,3 \mathrm{~mm}^{2}$ & $3,0 \%$ \\
\hline 3 & $22.1 \%$ & $38,8 \mathrm{~mm}^{2}$ & $1,4 \%$ \\
\hline
\end{tabular}

\section{Análisis de resultados}

Al comparar las figuras 1 y 2 , es notorio que la complejidad de la tarea computacional aumenta notablemente dado que, al presentarse más de una capa de tabletas, estas no se distribuyen de una manera uniforme ante la cámara. En cambio, aumenta la variabilidad de ángulo respecto al eje óptico en las tabletas, lo que produce efectos de sombras e iluminación no uniforme.

Los datos mostrados en el cuadro 1 demuestran que, a mayor cantidad de capas, la medición del área de las tabletas perpendiculares muestra ser más precisa en cuanto a la comparación con el área teórica. Este fenómeno podría justificarse basado en que se tiene una menor variabilidad e las mediciones dado que la muestra es menor, sumado al hecho de que, al aumentar las sombras los contornos de los objetos en la imagen se define mejor, facilitando la identificación de los mismos, Por otra parte, este fenómeno compromete los cálculos de las áreas de las tabletas rotadas, debido a que el fondo ya no es visible, por lo tanto, la distribución de pixeles por tono de gris se hace más homogénea y la umbralización pierde precisión. 
Por su parte, en la figura 4 se muestra la tendencia del número de tabletas perpendiculares en función del número de capas mostradas en el cuadro 1. Como puede verse, la variación en los porcentajes entre capas tiende a disminuir conforme aumenta el número de capas.

\section{Conclusiones}

Dados los datos obtenidos, se demuestra la eficacia del método para la obtención de datos experimentales, permitiendo obtener una primera aproximación al comportamiento del lecho de recubrimiento de tabletas en función del número de capas.

Debido a la naturaleza estocástica del acomodo de las tabletas en el lecho, es posible que, al aumentar la cantidad de capas, la afección de la cantidad de capas inferiores sobre la primera capa tienda a converger posterior a un número determinado de capas. Es decir, se plantea la hipótesis que la variación en la cantidad de tabletas perpendiculares a la cámara al pasar de un número determinado al siguiente será despreciable tras un número determinado de capas, pero este estudio sobrepasa las limitaciones propuestas para esta investigación y se plantea como trabajo futuro, evaluar el comportamiento de lechos con una mayor cantidad de capas de tabletas, así como el desarrollo de un modelo de simulación que tome en cuenta la posición de las mismas.

El tiempo de ejecución del algoritmo a priori podría presentar una limitante en cuanto a la ejecución en tiempo real del algoritmo de caracterización, no obstante, el tiempo de ejecución del algoritmo en una computadora diseñada para análisis de datos y dedicada únicamente al proceso será mucho menor. Asimismo, el algoritmo planteado podrá ser sujeto de optimizaciones posteriores, de modo que el tiempo de ejecución del algoritmo en tiempo real no comprometa la funcionabilidad del sistema de caracterización una vez implementado en un proceso real.

\section{Agradecimientos}

Se le agradece al Dr. Juan Luis Crespo Mariño coordinador del Laboratorio de Inteligencia Artificial para las Ciencias Naturales (LIANA), al Dr. Ernesto Montero Zeledón coordinador del Laboratorio Espectroscopía Molecular, Imagen y Color (LEMIC) del Instituto Tecnológico de Costa Rica (ITCR), por su constante apoyo y guía a lo largo de la presente investigación, y al Dr. Jorge Pacheco Molina el Instituto de Investigaciones Farmacéuticas (INIFAR) de la Facultad de Farmacia, de la Universidad de Costa Rica (UCR) por su apoyo en la fabricación de los comprimidos recubiertos.

\section{Referencias}

[1] M. Murillo, et. al. "Colour evolution during a coating process of pharmaceutical tablet cores by random spraying", Color Res Appl. Vol 44, 160-167, 2019.

[2] S. Muschert "Polymeric coatings for solid dosage forms: characterization and optimization", tesis de dorctorado, Universidad de Lille, Francia, 2008.

[3] FDA. Department of Health and Human Services U.S. Food and Drug Administration. "Guidance for Industry PAT - A Framework for Innovative Pharmaceutical Development, Manufacturing, and Quality Assurance". 2004.

[4] M. Murillo-Fernández, D. Gutiérrez-Fallas, J. Pacheco-Molina, \& E. Montero-Zeledón, "Physical and optical characterization of solid dosage forms coatings" presentado en Simposio en Ciencia de Materiales Avanzados y Nanotecnología, San José, Costa Rica, diciembre, 2016.

[5] F. Doerr et al. "Morphological Characterization of Solid Pharmaceutical Products using X-ray tomography", presentado en Micro-CT User Meeting 2017 Bruselas, BE, junio, 2017. 
[6] M. Murillo-Fernández, D. Gutiérrez-Fallas, J. Pacheco-Molina, \& E. Montero-Zeledón. "Caracterización de tabletas farmacéuticas recubiertas por el método de aspersión aleatoria", Tecnologías en Marcha, vol 32 Especial, 53-62, marzo 2019.

[7] M. Murillo-Fernández "Caracterización y modelización del recubrimiento de tabletas farmacéuticas, para el desarrollo de soluciones de reconocimiento óptico-visible", tesis de doctorado, Instituto Tecnológico de Costa Rica-Universidad de Costa Rica, 2020.

[8] A. J. Fitzgerald, B. E, Cole \& P. F. Taday "Nondestructive Analysis of Tablet Coating Thicknesses Using Terahertz Pulsed Imaging", Journal of Pharmaceutical Sciences, vol. 9(1), 177-183, 2005.

[9] O. V. García "La calidad desde el diseño: principios y oportunidades para la industria farmacéutica" Estudios Gerenciales, vol. 31, 68-78, 2015.

[10] D. Reitter. "FDA Draft Guidance Document Comments on Use of Inks, Pigments, Flavors and Taggants to Guard Against Counterfeiting". ColorCon Ing. 


\section{Anexos}

Imágenes utilizadas para las pruebas

Para los distintos análisis y calibración del algoritmo se utilizaron cinco imágenes para cada número de capas de tabletas, estas se muestran a continuación en las figuras A.1, A.2 y A.3.

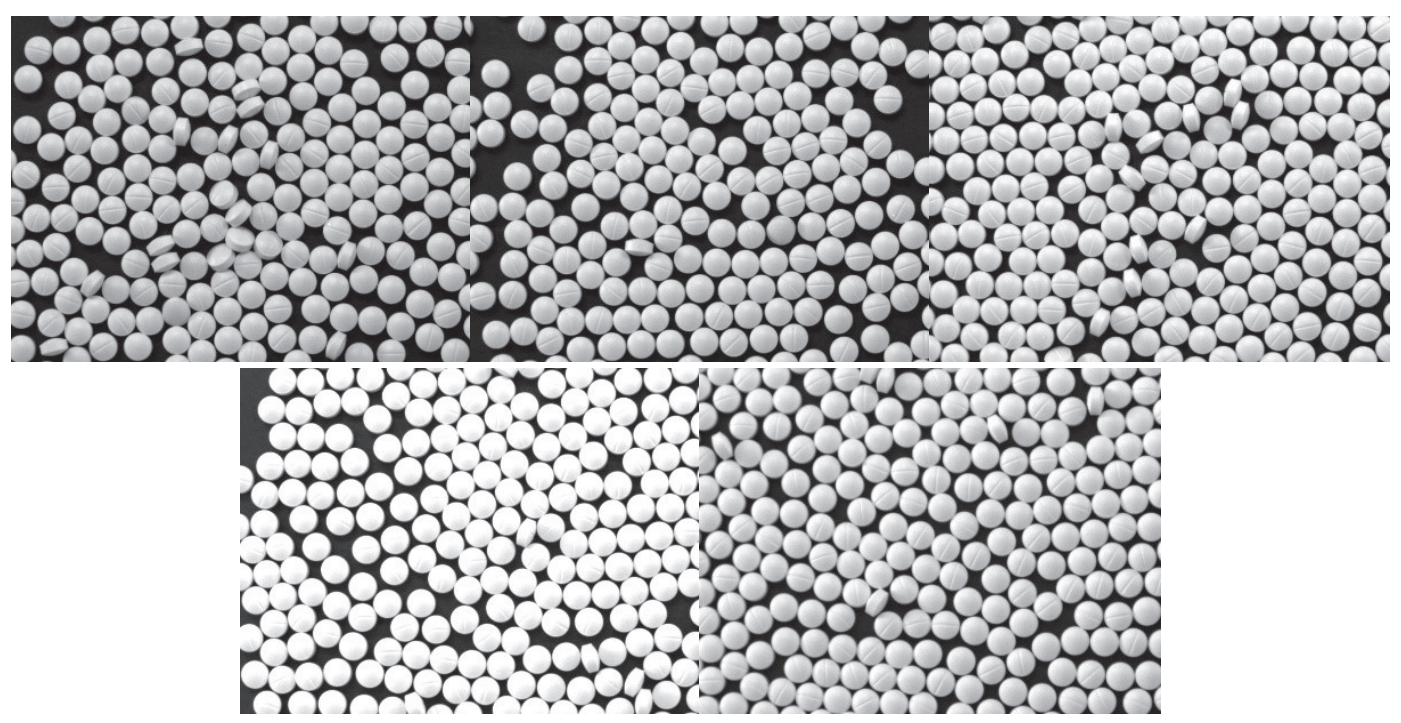

Figura A.1. Imagen de muestra 1, 2, 3, 4 y 5 con una capa de tabletas.

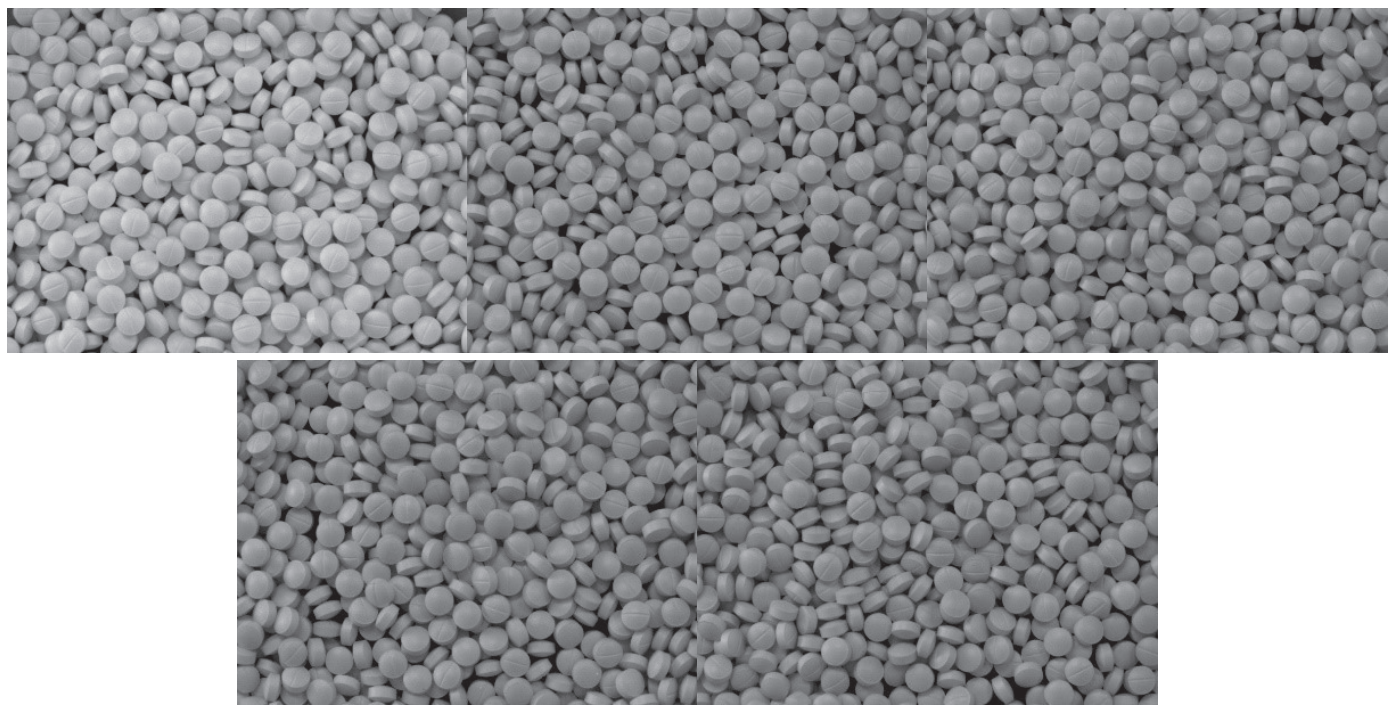

Figura A.2. Imagen de muestra 1, 2, 3, 4 y 5 con dos capas de tabletas. 

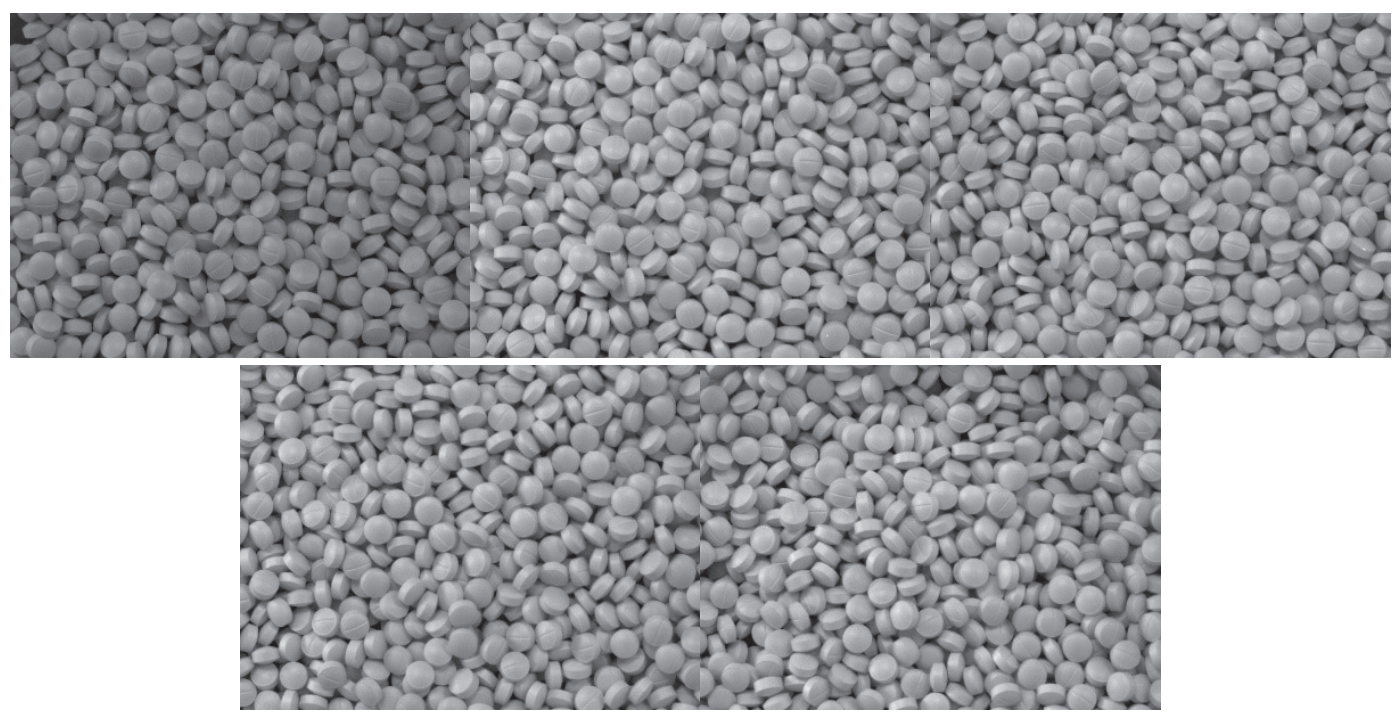

Figura A.3. Imagen de muestra 1, 2, 3, 4 y 5 con tres capas de tabletas.

Resultados obtenidos por capa

A continuación, en el cuadro A.2 se muestran la totalidad de los datos encontrados para cada una de las fotografías tomadas.

Cuadro A.2. Datos promedio obtenidos por capa.

\begin{tabular}{|c|c|c|c|c|c|}
\hline $\begin{array}{c}\text { Número } \\
\text { de capas }\end{array}$ & $\begin{array}{c}\text { Número de } \\
\text { muestra }\end{array}$ & $\begin{array}{c}\text { Tabletas } \\
\text { encontradas }\end{array}$ & $\begin{array}{c}\text { Tabletas } \\
\text { perpendiculares } \\
\text { encontradas }\end{array}$ & $\begin{array}{c}\text { Área de las tabletas } \\
\text { perpendiculares }\end{array}$ & $\begin{array}{c}\text { Porcentaje } \\
\text { de error }(\%)\end{array}$ \\
\hline 1 & 1 & 201 & 172 & 36,4 & 5,42 \\
\hline 1 & 2 & 190 & 168 & 36,3 & 5,68 \\
\hline 1 & 3 & 221 & 192 & 36,3 & 5,68 \\
\hline 1 & 4 & 207 & 183 & 36,4 & 5,42 \\
\hline 1 & 5 & 214 & 191 & 36,6 & 4,90 \\
\hline 2 & 1 & 210 & 85 & 37,6 & 4,30 \\
\hline 2 & 2 & 260 & 93 & 36,9 & 3,60 \\
\hline 2 & 3 & 240 & 88 & 37,1 & 2,56 \\
\hline 2 & 5 & 244 & 84 & 37,5 & 2,56 \\
\hline 3 & 1 & 227 & 83 & 38,1 & 1,00 \\
\hline 3 & 2 & 224 & 50 & 37,9 & 1,52 \\
\hline 3 & 3 & 225 & 57 & 37,7 & 2,04 \\
\hline 3 & 4 & 222 & 48 & 37,9 & 1,52 \\
\hline 3 & 5 & 211 & 44 & 38.8 & 0.8 \\
\hline
\end{tabular}

